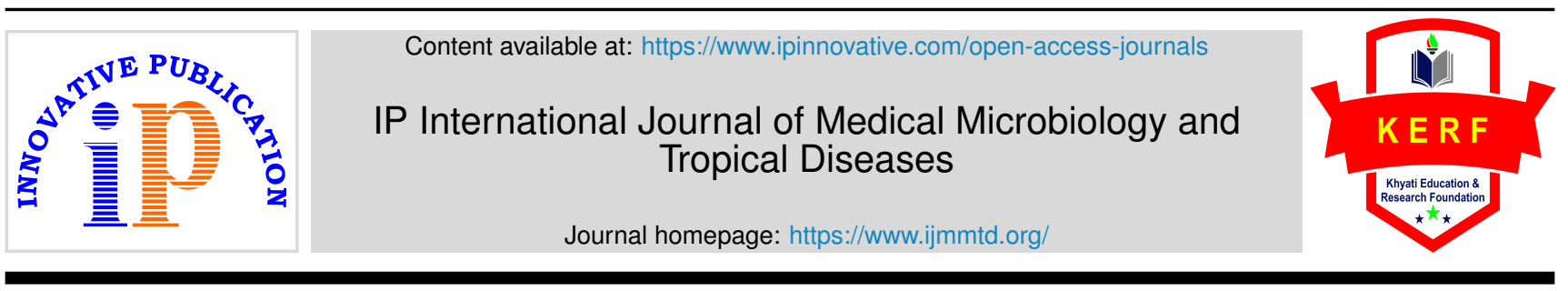

Original Research Article

\title{
Transformation of commensal to a pathogen: Blood stream infection due to coagulase negative staphylococci among patients attending tertiary care hospital in North India
}

\author{
Vikramjeet Singh ${ }^{1}$, Pranshu Pandey ${ }^{1}$, Anupam Das ${ }^{1}$ *, Manodeep Sen ${ }^{1}$, \\ Jyotsna Agarwal ${ }^{1}$ \\ ${ }^{1}$ Dept. of Microbiology, Dr. Ram Manohar Lohia Institute of Medical Sciences, Lucknow, Uttar Pradesh India, India
}

\section{A R T I C L E I N F O}

Article history:

Received 10-05-2021

Accepted 25-05-2021

Available online 25-06-2021

\section{Keywords:}

Coagulase Negative Staphylococcus

Commensal

Blood stream infection

Antimicrobial resistance

\begin{abstract}
A B S T R A C T
Aims and Objectives: Aim of this study was to know the prevalence of Coagulase Negative Staphylococci (CoNS) in Blood Stream Infection (BSI) among patients attending a Superspeciality hospital in North India. Objective of this study was to compare incidence of CoNS in ICU and ward patients suffering from BSI and their antimicrobial susceptibility pattern.

Materials and Methods: This retrospective hospital based study was conducted in the Microbiology Department, Dr. Ram Manohar Lohia Institute of Medical Sciences, Lucknow for a period of two years (January, 2017 -December, 2018). Blood samples from a total of 6498 patients from Out-Patient, In-Patient and Intensive care unit departments were subjected to aerobic and anaerobic bacterial culture. Culture positive broth was subcultured on Blood Agar and MacConkey Agar to isolate pathogens in pure culture. Pure cultured isolates were tested for antimicrobial susceptibility pattern by Kirby Bauer Disk Diffusion method as per CLSI 2018.

Results: During the study period, out of total 3284 samples in 2017 and 3214 in 2018, 663 and 595 were found to be culture positive respectively. Staphylococci were isolated from $636(9.78 \%)$ patients $(10.2 \%$ in 2017 and 9.2\% in 2018). Staphylococcus aureus was isolated from $18.8 \%$ and CoNS from $81.2 \%$ of total Staphylococcal isolates. Among CoNS isolates $70.7 \%$ were found to be Methicillin Resistant CoNS (MRCoNS).

Discussion and Conclusion: This study observed CoNS as a major cause of BSI as compared to Staphylococcus aureus. The significance of CONS bacteremia should be evaluated better in light of clinical profile of the patient. Better screening and infection control practices in the future can decrease the rate of methicillin resistant CoNS in our centre.

(C) This is an open access article distributed under the terms of the Creative Commons Attribution License (https://creativecommons.org/licenses/by/4.0/) which permits unrestricted use, distribution, and reproduction in any medium, provided the original author and source are credited.
\end{abstract}

\section{Introduction}

Blood stream infection (BSI) is a potentially life threatening infection, associated with high mortality and morbidity. Coagulase negative Staphylococci (CoNS) and Staphylococcus aureus are the most commonly isolated Gram positive bacteria from blood culture samples. S. aureus is a known pathogen causing BSIs, while CoNS were considered to be common contaminants of blood culture.

\footnotetext{
* Corresponding author.

E-mail address: dasdranupam@gmail.com (A. Das).
}

CoNS are found to be the most frequent cause of nosocomial bloodstream infections. ${ }^{1}$ CoNS are a part of the normal microflora of skin but can also colonize the nasal mucosa, the lower airways and invasive devices. ${ }^{2}$ In clinical practice, it is important to distinguish between contamination and BSI to prevent unnecessary prescription of antimicrobial agents leading to a selection of antimicrobial-resistant organisms, longer hospitalization and increased costs. ${ }^{3}$ Mainly due to lack of a diagnostic reference standard for BSI, the clinical relevance of a single blood culture positive for CoNS is difficult to assess. ${ }^{4}$ True BSI is 
considered when at least 2 blood culture yield CoNS whereas contamination is assumed if only one of the two sets of blood cultures shows growth of CoNS. ${ }^{5}$ However, several studies reported that about one-third of patients with true BSI had only one positive blood culture and that contamination was possible even if two or more sets were positive. Recently many studies have challenged this traditional viewpoint, showing an increased recognition of CoNS as agents causing BSI. Resistance to antimicrobials in CoNS is increasing, especially the frequency of methicillinresistant CoNS (MRCoNS) in hospitalized patients. ${ }^{6}$

Aim of this study was to determine the frequency of Coagulase Negative Staphylococci (CoNS) in Blood Stream Infection (BSI) among patients presenting to a tertiary health care super speciality Institute in North India. Objective of this study was to compare frequency of CoNS in ICU, IPD, OPD (of tertiary care hospital) and referral centres (outside the hospital) patients suffering from BSI including their antimicrobial susceptibility patterns.

\section{Materials and Methods}

This retrospective hospital based study was conducted in the Department of Microbiology, Dr. Ram Manohar Lohia Institute of Medical Sciences, Lucknow for a period of two years (January 2017-December 2018). After skin preparation with $70 \%$ alcohol and 2\% iodine solution, 5-10 $\mathrm{ml}$ blood was collected by venepuncture and inoculated into blood culture bottles containing $50 \mathrm{ml}$ brain heart infusion broth with sodium polyanetholesulphonate as an anticoagulant. On receiving blood samples from a total of 6498 patients from OPD, IPD and ICU departments were subjected to aerobic and anaerobic bacteria culture. Culture positive broth was subcultured on Blood Agar and MacConkey Agar to isolate different pathogens in pure culture. Pure cultured isolates were identified by Gram's staining and conventional biochemical. ${ }^{7}$ Further differentiation of Staphylococcal isolates into Staphylococcus aureus and CoNS was done by using Deoxy- Ribonuclease agar, slide coagulase and tube coagulase tests. Staphylococcus aureus and CoNS isolates were subjected to antimicrobial susceptibility testing (AST) on Mueller-Hinton agar with the following antibiotic discs (Oxoid and Sigma aldrich Laboratories) using the Kirby Bauer disc diffusion method: penicillin (10 U), cefoxitin (30 $\mu \mathrm{g})$, Erythromycin $(15 \mu \mathrm{g})$, Gentamicin $(10 \mu \mathrm{g})$, Tetracycline $(30 \mu \mathrm{g})$, Doxycycline $(30 \mu \mathrm{g})$ Ciprofloxacin $(5$ $\mu \mathrm{g})$, Levofloxacin $(5 \mu \mathrm{g})$, Clindamycin $(2 \mu \mathrm{g})$, Linezolid $(30 \mu \mathrm{g})$ and Chloramphenicol $(30 \mu \mathrm{g})$. An oxacillin disc (1 $\mu \mathrm{g})$ was used to detect methicillin resistance. Sensitivity was read after incubation for $24 \mathrm{hrs}$ at $35+2^{\circ} \mathrm{C}$. Isolates were regarded as sensitive, intermediate or resistant according to Clinical and Laboratory Standards Institute (CLSI) 2018 guidelines. ${ }^{8,9}$ AST patterns of isolates from ICU, IPD, OPD and from other referral centers of Lucknow were analyzed. The data generated in this study were subjected for statistical analysis with the help of appropriate bio-statistical tool (SPSS 16.0 Software for windows) for interpretation of significant outcome.

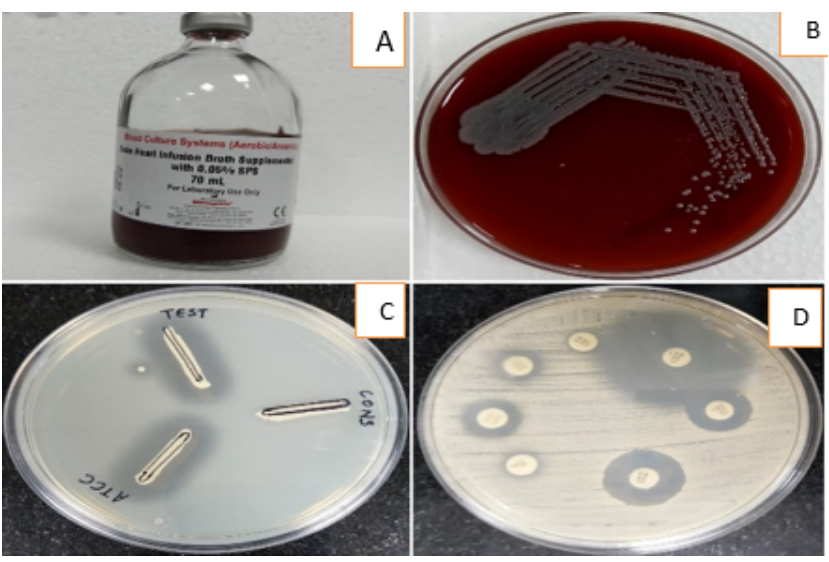

Fig. 1: A- Blood culture bottle; B- S. aureus on Blood agar; CDNase Test; D- AST pattern of Staphylococcus

\section{Result}

During the study period, out of total 3284 samples in 2017 and 3214 in 2018, $663(20.2 \%)$ and $595(18.5 \%)$ were found to be culture positive respectively. All clinically suspected patients whether hospitalized or OPD of bacteremia were included in the present study. Of these, 35 patients were excluded (10 in 2017 and 25 in 2018) because of contamination during blood culture collection. The average patient age was $56+10$ years; $58 \%$ were male and $42 \%$ were female. This finding was in accordance with Elzi et al. (2012) (60\%), Raad et al. (2009) (63\%) and Rewa et al. (2012) (54.8\%). ${ }^{10-12}$ Amongst the isolated bacterial pathogens gram positive cocci were the predominant ones, 402 and 360 isolates in 2017 and 2018 respectively. Staphylococci were isolated from 636 (9.78\%) patients (10.2\% in 2017 and $9.2 \%$ in 2018). Staphylococcus aureus was isolated from $18.8 \%$ and CoNS from $81.2 \%$ of total Staphylococcal isolates. In comparison to 2017, there was decrease in the incidence of Coagulase negative Staphylococcus spp. in 2018 notably in the department of OPD, IPD and ICU. However, there was surge in the incidence of CoNS BSI in referral centre by $2 \%$. There was no significant change in the incidence of BSI due to Staphylococcus aureus in 2017 to 2018.

In the present study we did not find any significant difference among the frequency of CoNS as well as S.aureus among the patients attending OPD $(\mathrm{p}=0.06 ; \mathrm{p}=0.32)$, Referral centres $(\mathrm{p}=0.7 ; \mathrm{p}=0.7)$ or IPD $(\mathrm{p}=0.06 ; \mathrm{p}=0.33)$ in 2017 and in 2018. However, we observed significant difference in the frequency of CoNS $(\mathrm{p}=0.002)$ in Intensive care unit patients, there was considerable decrease in the 
Table 1: Distribution of Coagulase negative staphylococcus and Staphylococcus aureus in BSI in various departments

\begin{tabular}{|c|c|c|c|c|}
\hline \multirow{2}{*}{$\begin{array}{l}\text { Positive Samples in } \\
\text { various Departments }\end{array}$} & \multicolumn{2}{|c|}{2017} & \multicolumn{2}{|c|}{2018} \\
\hline & CoNS $(\%)$ & $\begin{array}{c}\text { Staphylococcus aureus } \\
(\%)\end{array}$ & CoNS $(\%)$ & $\begin{array}{c}\text { Staphylococcus aureus } \\
(\%)\end{array}$ \\
\hline OPD & 32 / 67 (47.8\%) & $8 / 67(11.9 \%)$ & 17 / $61(\mathbf{2 7 . 9 \% )}$ & $4 / 61(6.6 \%)$ \\
\hline IPD & $94 / 215(43.7 \%)$ & $15 / 215(7 \%)$ & 14 / $54(\mathbf{2 5 . 9 \%})$ & $6 / 54(\mathbf{1 1 \%})$ \\
\hline ICU & $39 / 156(\mathbf{2 5 \%})$ & $6 / 156(3.8 \%)$ & $12 / 126(\mathbf{9 . 5 \%})$ & $5 / 126(3.8 \%)$ \\
\hline Referral Centres & $119 / 231(\mathbf{5 2 \%})$ & $28 / 231(\mathbf{1 2 \%})$ & $190 / 354(\mathbf{5 4 \%})$ & 47 / $354(\mathbf{1 3 \%})$ \\
\hline Total & 284 / $669(\mathbf{4 2 . 5 \%})$ & 57 / $669(8.5 \%)$ & 233 / 595 (39.1\%) & 62 / $595(\mathbf{1 0 . 4 \% )}$ \\
\hline
\end{tabular}

incidence of Coagulase negative staphylococcus isolates.

Among the 517 isolates, a $59.7 \%$ prevalence of MRCoNS was seen. An overall high prevalence of resistance to the non- $\beta$-lactam antimicrobials was observed including clindamycin, gentamicin, erythromycin and fluoroquinolone like ciprofloxacin and levofloxacin. All the isolates were uniformly susceptible to vancomycin, teicoplanin and linezolid. The MRCoNS showed a higher level of resistance to all non- $\beta$-lactam antimicrobials tested as compared to MSCoNS [Table 2]. The main public health problem associated with high mortality, longer hospitalization, and exorbitant treatment cost is antibiotic resistance. For effective analysis of bacteremia, the collection of blood cultures should precede empirical treatment and modified based on antibiotic susceptibility and resistance pattern. MRCoNS showed higher level of resistance to all non- $\beta$-lactam antimicrobials compared to MSCoNS with difference being statistically significant for Fluoroquinolones (63\% vs $18 \%$; p value $<0.0001,{ }^{2}$ value $=79.11)$, aminoglycosides $(51 \%$ vs $20 \%$; p value $<0.001,{ }^{2}$ value $\left.=38.275\right)$ and macrolides antibiotics like erythromycin $\left(74 \%\right.$ vs $28 \%$; p value $<0.001 ;^{2}$ value $=$ 88.32).

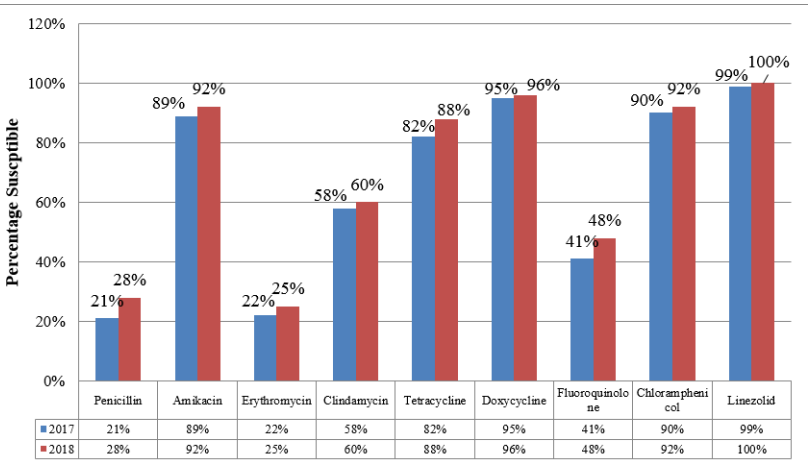

Graph 1: CoNS antimicrobial susceptibility in OPD BSI cases in 2017 and 2018

MDR was present in $41.6 \%$ of the CoNS isolates $(\mathrm{n}=$ $215)$. Among the MRCoNS, MDR was found in $69.4 \%$. This finding was in accordance with study conducted by Kumhar et al. (2002) and Jain et al. (2004) in North India. ${ }^{13,14}$ Maximum number of MDR CoNS isolates were recovered

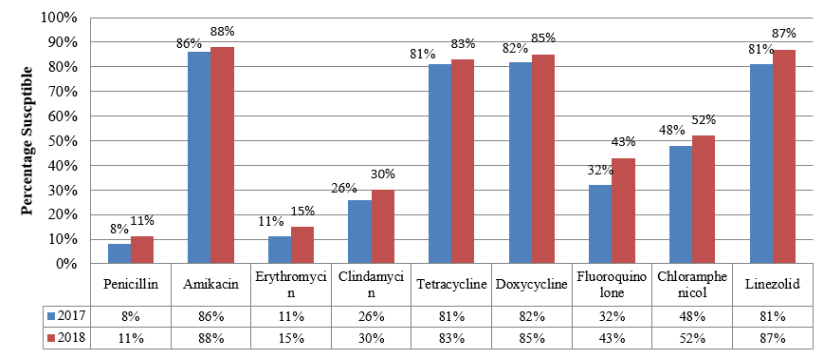

Graph 2: CoNS antimicrobial susceptibility in ICU BSI cases in 2017 and 2018

from patients admitted to ICUs (85.67\%) and minimum from those admitted to OPD and referral centres (28.2\%). There was a significant difference $(p$ value $<0.05$ ) among the isolates of MRCoNS isolated from ICU and IPD in 2017, However there was no statistical significance among MRCoNS isolates of ICU and IPD in 2018.

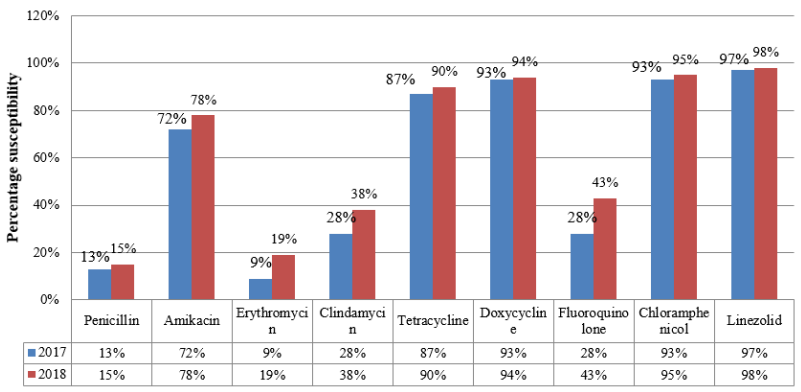

Graph 3: CoNS antimicrobial susceptibility in IPD BSI cases in 2017 and 2018

\section{Discussion}

Two decades back, Coagulase-negative Staphylococci were considered as contaminants of bacterial cultures. However, this group especially $\mathrm{S}$. epidermidis has emerged as an important pathogen and a major cause of serious infections in ICU patients. In this study, S. epidermidis was the most common isolated member of CoNS. This was in agreement with prior reports conducted in adult ICUs and similar results have been shown by Usha MG et al. (2013) who 
Table 2: Comparison of antimicrobial susceptibility in MRCoNS and MSCoNS

\begin{tabular}{|c|c|c|c|c|c|}
\hline \multirow{2}{*}{$\begin{array}{l}\text { Antimicrobials } \\
\text { Tested }\end{array}$} & \multicolumn{2}{|c|}{ MRCoNS Susceptibilty } & \multicolumn{2}{|c|}{ MSCoNS Susceptibility } & \multirow{2}{*}{ P Value } \\
\hline & $2017(n=217)$ & $2018(n=163)$ & $2017(n=67)$ & $2018(n=70)$ & \\
\hline Ciprofloxacin & $68(31 \%)$ & $72(44 \%)$ & $45(67 \%)$ & $56(80 \%)$ & $\mathrm{P}<0.001$ \\
\hline Levofloxacin & $103(48 \%)$ & $92(57 \%)$ & $52(78 \%)$ & $61(87 \%)$ & $\mathrm{P}<0.001$ \\
\hline Gentamicin & $99(46 \%)$ & $86(53 \%)$ & $53(79 \%)$ & $57(81.5 \%)$ & $\mathrm{P}<0.001$ \\
\hline Erythromycin & $48(22 \%)$ & $52(32 \%)$ & $47(70 \%)$ & $52(74 \%)$ & $\mathrm{P}<0.001$ \\
\hline Clindamycin & $67(31 \%)$ & $61(37 \%)$ & $51(76 \%)$ & $58(83 \%)$ & $\mathrm{P}<0.001$ \\
\hline Tetracycline & $153(71 \%)$ & $124(76 \%)$ & $63(94 \%)$ & $63(90 \%)$ & $\mathrm{P}<0.001$ \\
\hline
\end{tabular}

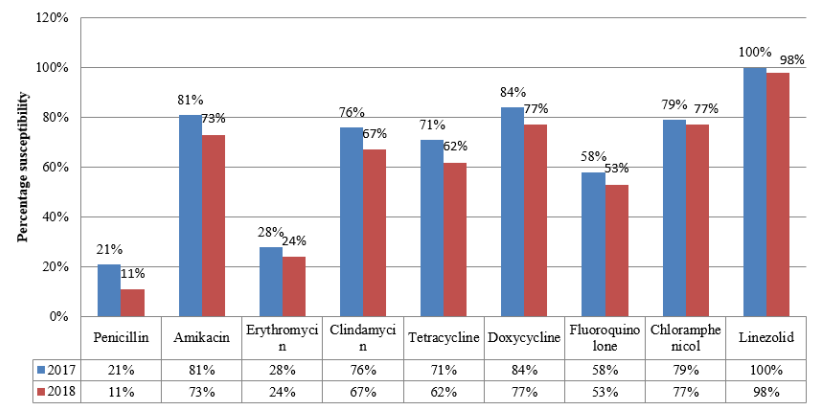

Graph 4: CoNS antimicrobial susceptibility in referral centres outside Hospital BSI cases in 2017 and 2018

studied the epidemiology from blood, pus, and urine isolates and showed that $\mathrm{S}$. epidermidis was the most common isolate followed by S. haemolyticus and S. lugdunensis. ${ }^{15,16}$

The prevalence of MRCoNS in our study ranged from $76 \%$ in 2017 to $70 \%$ in 2018 among total CoNS isolates. On PubMed search for Indian study on antibiotics resistance pattern of different CoNS species during the last 10 years, two studies were found - Asangi et al., 2011 and Singh S et al., 2016., ${ }^{17,18}$ Both the authors showed similar resistance high resistance to penicillin for Coagulase negative Staphylococcus; similar to our study. The high prevalence of MRCoNS not only decreases the treatment options and also assists in transfer of the resistance elements to other staphylococcal strains present in the hospital settings. The presence of methicillin resistance in CoNS has the potential or the ability to transfer resistance mechanisms to other virulent pathogens such as Staphylococcus aureus present on the skin and environment. The rise of MRCoNS was higher in ICU patients because of the presence of comorbid conditions, parenteral nutrition and drug administration using intravascular devices.

Similar findings were observed in another study from India on neonatal septicaemia (Kumhar et al., 2002), although in that study, CNS was not a major isolate. Penicillin resistance was frequent (94\%), while amikacin resistance was relatively rare $(19 \%)$. Resistance to antibiotics was seen more in the methicillin-resistant isolates compared with those that were methicillin sensitive.

The significance of CONS bacteremia should be evaluated better in light of clinical profile of the patient.
High rates of methicillin resistance in our center in 2017 called for a better screening and infection control practices in 2018, which led to increase in susceptibility percentage of many antimicrobials in positive blood culture samples coming from ICU, IPD and OPD. However, this was not the case with the samples coming from referral centers as no health education or sample collection training was conducted at these centers as a result of which we observed rising trends of antimicrobial resistance in referral centers in the year 2018 also.
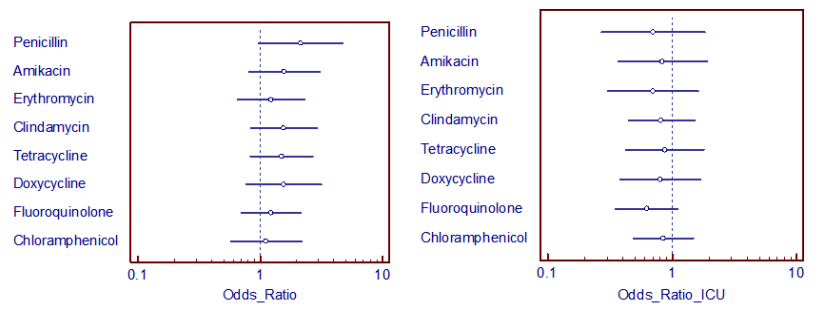

Graph 5: Odds Ratio (A) referral centres and (B) ICU

The most important factor for preventing nosocomial colonization and infections in an hospital is to practice hand hygiene in a best possible manner. It has been observed that the compliance to hand disinfection is often poor in OPD and referral centres due to inadequate health education programmes, in comparison to ICU and wards where monthly health education programme is conducted for best infection prevention practices, being lowest for the physicians working at OPD and outskirts settings, which further exposes these immunocompromised patients to colonization of resistant organisms like CoNS leading to BSI.

\section{Conclusion}

CoNS should be regarded as pathogen of significance and not as contaminant only. Various species such as S. epidermidis, S. haemolyticus, and S. lugdunensis are causing infections these days. MRCoNS prevalence is also increasing and was in the range of $50 \%-68.67 \%$ for various species in this study. $100 \%$ susceptibility was seen towards vancomycin, teicoplanin, and linezolid. Still these drugs should be used as reserve drugs. To prevent CoNS infections, hospital infection control measures should be 
Table 3: Comparison between Referral centres (Outside hospital) and ICU regarding antimicrobial susceptibility in CoNS BSI cases.

\begin{tabular}{|c|c|c|c|c|c|c|c|c|c|c|}
\hline \multirow[t]{2}{*}{ Antimicrobials } & \multicolumn{2}{|c|}{$\begin{array}{c}\text { Referral } \\
\text { Centres } \\
\text { (Susceptible } \\
\text { Percentage) }\end{array}$} & \multirow[t]{2}{*}{$\begin{array}{l}\text { Odds } \\
\text { Ratio }\end{array}$} & \multicolumn{2}{|c|}{$\begin{array}{l}\text { 95\% Confidence } \\
\text { Interval }\end{array}$} & \multicolumn{2}{|c|}{$\begin{array}{l}\text { ICU (Susceptible } \\
\text { percentage) }\end{array}$} & \multirow[t]{2}{*}{$\begin{array}{l}\text { Odds } \\
\text { Ratio }\end{array}$} & \multicolumn{2}{|c|}{$\begin{array}{l}\text { 95\% Confidence } \\
\text { Interval }\end{array}$} \\
\hline & 2017 & 2018 & & Low & High & 2017 & 2018 & & Low & High \\
\hline Penicillin & 21 & 11 & 2.15 & 0.976 & 4.73 & 8 & 11 & 0.7 & 0.27 & 1.83 \\
\hline Amikacin & 81 & 73 & 1.57 & 0.802 & 3.07 & 86 & 88 & 0.83 & 0.367 & 1.914 \\
\hline Erythromycin & 28 & 24 & 1.23 & 0.653 & 2.32 & 11 & 15 & 0.7 & 0.305 & 1.61 \\
\hline Clindamycin & 76 & 67 & 1.55 & 0.839 & 2.89 & 26 & 30 & 0.81 & 0.442 & 1.522 \\
\hline Tetracycline & 71 & 62 & 1.5 & 0.831 & 2.71 & 81 & 83 & 0.87 & 0.424 & 1.798 \\
\hline Doxycycline & 84 & 77 & 1.56 & 0.771 & 3.18 & 82 & 85 & 0.8 & 0.382 & 1.701 \\
\hline Fluoroquinolone & 58 & 53 & 1.23 & 0.701 & 2.14 & 32 & 43 & 0.62 & 0.351 & 1.11 \\
\hline Chloramphenicol & 179 & 77 & 1.12 & 0.575 & 2.19 & 48 & 52 & 0.85 & 0.489 & 1.484 \\
\hline
\end{tabular}

strengthened, and staff should be instructed to follow hand hygiene practice. Speciation of CoNS and its antibiotic susceptibility pattern will guide the clinicians in establishing their role as significant pathogens and initiate proper antimicrobial therapy based on the susceptibility pattern.

\section{Conflicts of Interest}

All contributing authors declare no conflicts of interest.

\section{Source of Funding}

None

\section{References}

1. Rogers KL, Fey PD, Rupp ME. Coagulase-Negative Staphylococcal Infections. Infect Dis Clin North Am . 2009;23(1):73-98. d01:10.1016/].1dc.2008.10.001

2. Weinstein MP. Blood Culture Contamination: Persisting Problems and Partial Progress. J Clin Microbiol. 2003;41(6):2275-8. doi:10.1128/jcm.41.6.2275-2278.2003.

3. Seybold U, Reichardt C, Halvosa JS, Blumberg HM. Clonal Diversity in Episodes with Multiple Coagulase-Negative Staphylococcus Bloodstream Isolates Suggesting Frequent Contamination. Infection.

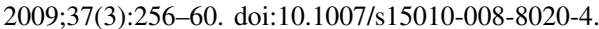

4. Bjorkqvist M, Soderquist B, Tornqvist E, Sjoberg L, Fredlund H, Kuhn I, et al. Phenotypic and genotypic characterisation of blood isolates of coagulase-negative staphylococci in the newborn. APMIS. 2002;110(4):332-9. doi:10.1034/].1600-0463.2002.100408.x.

5. Toldos CM, Yagüe G, Ortiz G, Segovia M. Assessment of multiple coagulase-negative staphylococci isolated in blood cultures using pulsed-field gel electrophoresis. Eur J Clin Microbiol Infect Dis . 1997;16(8):581-6. 101:010016+0244-1920.

6. Souvenir D, Anderson DE, Palpant S, Mroch H, Askin S, Anderson J, et al. Blood Cultures Positive for Coagulase-Negative Staphylococci: Antisepsis, Pseudobacteremia, and Therapy of Patients. $J$ Clin Microbiol . 1998;36(7):1923-6. [01:10.1128//cm.36.7.1923 प्य26.

7. Collee JG, Duguid JP, Fraser AG, Marmion BP, Simmon A. Laboratory strategy in diagnosis of infective syndromes. In: Mackie and McCartney Practical Medical Microbiology. 14th Edn.. vol. 2. London: Churchill Livingstone; 1996. p. 245-61.

8. Baird D. Staphylococcus: Cluster forming gram positive cocci. In: Collee J, Fraser A, Marmion B, Simmons A, editors. Mackie and McCartney Practical Medical Microbiology. vol. 2. London: Churchill Livingstone; 1996. p. 245-61.
9. Performance Standards for Antimicrobial Susceptibility Testing. 28th Edn. In: CLSI supplement M100. Wayne, PA: Clinical and Laboratory Standards Institute; 2018.

10. Elzi L, Babouee B, Vögeli N, Laffer R, Dangel M, Frei R, et al. How to discriminate contamination from bloodstream infection due to coagulase-negative staphylococci: a prospective study with 654 patients. Clin Microbiol Infect. 2012;18(9):E355-61. doi:10.111/1/.1469-0691.2012.03964.x

11. Raad I, Kassar R, Ghannam D, Chaftari AM, Hachem R, Jiang Y, et al. Management of the Catheter in Documented Catheter-Related Coagulase-Negative Staphylococcal Bacteremia: Remove or Retain? Clin Infect Dis. 2009;49(8):1187-94. 101:10.1086/605694

12. Rewa O, Muscedere J, Reynolds S, Jiang X, Heyland DK. CoagulaseNegativeStaphylococcus, Catheter-Related, Bloodstream Infections and their Association with Acute Phase Markers of Inflammation in the Intensive Care Unit: An Observational Study. Can J Infect Dis Med Microbiol. 2012;23(4):204-8. [0i:10/155/2012/1983831.

13. Kumhar GD, Ramachandran VG, Gupta P. Bacteriological analysis of blood culture isolates from neonates in a tertiary care hospital in India. J Health Popul Nutr. 2002;20:343-7.

14. Jain A, Agarwal J, Bansal S. Prevalence of methicillin-resistant, coagulase-negative staphylococci in neonatal intensive care units: findings from a tertiary care hospital in India. J Med Microbiol . 2004;53(9):941-4. doi:10.1099/jmm.0.45565-0

15. Gautam V, Sethuraman N, Kaur R, Sachdev S, Marwaha N, Ray P, et al. Changing Epidemiology of Coagulase-Negative Staphylococci in Normal Flora of Skin. Indian J Med Microbiol . 2017;35(2):277-8. [01:10.4103/1] mm.1]mm_16_282.

16. Usha MG, Shwetha DC, Vishwanath G. Speciation of coagulase negative Staphylococcal isolates from clinically significant specimens and their antibiogram. Indian J Pathol Microbiol . 2013;56(3):258-60. 10i: $10.4103 / 0377-4929.120383$.

17. Asangi SY, Mariraj J, Sathyanarayan MS, Nagabhushan. Speciation of clinically significant coagulase negative staphylococci and their antibiotic resistant pattern in a tertiary care hospital. Int J Biol Med Res. 2011;2:735-9.

18. Singh L, Cariappa MP, Das NK. Drug sensitivity pattern of various Staphylococcus species isolated at a tertiary care hospital. $\quad$ Med J Armed Forces India . 2016;72:S62-6. 001:10.1016/1.m]an.2016.07.009

\section{Author biography}

Vikramjeet Singh, Assistant Professor

Pranshu Pandey, Junior Resident

Anupam Das, Professor (Jr. Grade) 
Manodeep Sen, Professor

Jyotsna Agarwal, Professor \& Head
Cite this article: Singh V, Pandey P, Das A, Sen M, Agarwal J.

Transformation of commensal to a pathogen: Blood stream infection due to coagulase negative staphylococci among patients attending tertiary care hospital in North India. IP Int J Med Microbiol Trop Dis 2021;7(2):71-76. 